\title{
Comparison of Intensity Modulated Radiotherapy and Dynamic Three-Dimensional Conformal Radiotherapy With Regard to Dose Distribution and Sparing of Organs at Risk
}

\author{
Yuzo HaSegawa, ${ }^{1}$ Toshihiko IUCHI, ${ }^{1}$ Katsunobu OSATO, ${ }^{1}$ \\ Takashi KODAMA, ${ }^{2}$ Naoki TOYAMA, ${ }^{2}$ and Kazuo HATANO ${ }^{2}$
}

Divisions of ${ }^{1}$ Neurological Surgery and ${ }^{2}$ Radiation Oncology, Chiba Cancer Center, Chiba

\begin{abstract}
Dose escalation to the target while sparing the organs at risk near the lesion has been difficult over the last decade. However, recent radiotherapy techniques can deliver more sophisticated doses to the target. This study evaluated whether intensity modulated radiotherapy can deliver more homogeneous and conformal doses to the target than dynamic three-dimensional conformal radiotherapy while sparing organs at risk near the lesion in 13 patients with central nervous system tumors and other tumors around the central nervous system. Dynamic three-dimensional conformal radiotherapy and intensity modulated radiotherapy plans were calculated and dose distributions were compared for all patients with regard to the planning target volume and organs at risk. The plan of intensity modulated radiotherapy was significantly superior to that of dynamic three-dimensional conformal radiotherapy in target dose conformity $(p=\mathbf{0 . 0 0 0 6})$ and organs at risk sparing $(p=\mathbf{0 . 0 2 5 7})$. Intensity modulated radiotherapy could deliver more homogeneous and conformal doses to the target than dynamic three-dimensional conformal radiotherapy with sparing organs at risk near the lesion and may improve local control of radioresistant tumors via dose escalation.
\end{abstract}

Key words: intensity modulated radiotherapy, conformity, dose distribution, organ at risk, dose escalation

\section{Introduction}

The goal of radiotherapy is to treat patients with the best therapeutic ratio that provides the highest local control and the lowest toxicity rates. Dose escalation to the lesion improves local control of the tumors, ${ }^{8,12}$ but also increases the risk of normal tissue complication. Therefore, radiation dose distributions should be designed conforming to the entire lesion while sparing the surrounding normal tissues, especially organs at risk (OARs) which are radiosensitive and related to late complications, such as cataract of the eye lens.

Recent advancements in radiotherapy technologies have allowed sophisticated radiation dose delivery, including the use of dynamic three-dimensional conformal radiotherapy (3D-CRT) and intensity modulated radiotherapy (IMRT). ${ }^{1,17)}$ Both dy-

Received November 22, 2010; Accepted February 15, 2011 namic 3D-CRT and IMRT are linear accelerator therapies using the multileaf collimator (MLC), a computer-controlled device that uses movable "leaves" to conform the radiation beam to the shape of the tumor while protecting normal adjacent tissue. Dynamic 3D-CRT stereotactically delivers doses which conform to the shape of the each projected target using the MLC. IMRT can also use MLC but the shapes are not restricted to the projected images. In IMRT, the fixed radiation beam from each angle can be turned off and on, or set to deliver dose at different intensities freely. Therefore, the plans of IMRT are highly complicated so inverse-planning is usually selected. The planner defines targets and OARs, and gives target doses. Then, the most suitable treatment plan is calculated automatically (Fig. 1).

The present study tried to evaluate whether IMRT can deliver more homogeneous and conformal dose distributions to irregular shaped tumors than dynamic 3D-CRT with sparing of OARs near lesions in 


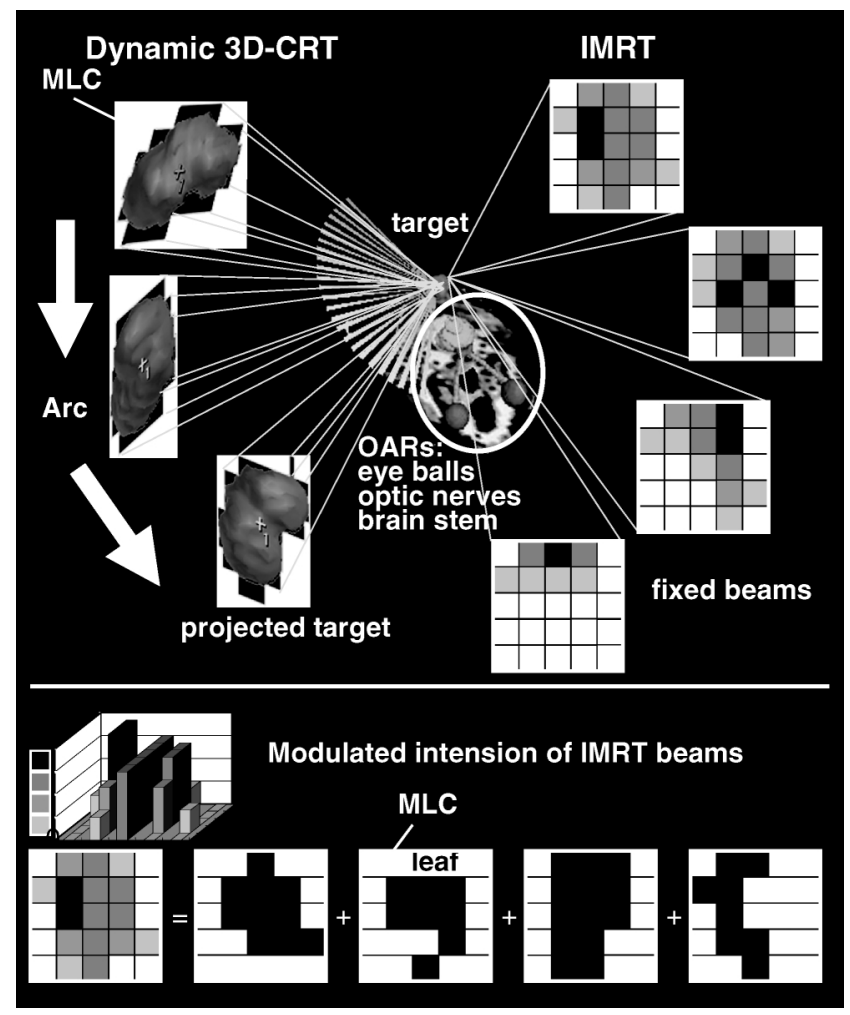

Fig. 1 Features of dynamic three-dimensional conformal radiotherapy (3D-CRT) and intensity modulated radiotherapy (IMRT). Dynamic 3D-CRT stereotactically delivers doses which conform to the shape of the each projected target using multileaf collimator (MLC). IMRT also uses MLC but not stereotactic technologies. The most suitable treatment plan using intensity modulated fixed beams is inversely calculated automatically. Arc: gantry arc, OARs: organs at risk.

the central nervous system (CNS) and other tumors around the CNS. Various tumors have been already treated with these techniques ${ }^{5,6,8,10,13,18,20)}$ and recent studies compared the dose conformity of dynamic 3D-CRT with IMRT ${ }^{7,9,14,19)}$ but little attention has focused on the OARs, especially in the CNS. ${ }^{3)}$ Patients with CNS tumors and other tumors around the CNS were enrolled in this study because these tumors were very close to the OARs. We compared dynamic 3D-CRT with IMRT focusing on target dose homogeneity, target dose conformity, and mean dose to the OARs.

\section{Patients and Methods}

Thirteen patients, 8 males and 5 females aged 8-69 years (median 48 years), with 9 skull base tumors, 1 brain stem tumor, and 3 vertebral body tumors were randomly enrolled (Table 1). Both computed tomo-
Table 1 Thirteen patients with central nervous system tumors

\begin{tabular}{|c|c|c|c|c|}
\hline $\begin{array}{l}\text { Case } \\
\text { No. }\end{array}$ & $\begin{array}{l}\text { Age } \\
\text { (yrs) }\end{array}$ & Sex & Lesion & Histology \\
\hline 1 & 45 & $\mathrm{M}$ & skull base & squamous cell carcinoma \\
\hline 2 & 62 & M & skull base & squamous cell carcinoma \\
\hline 3 & 62 & M & skull base & squamous cell carcinoma \\
\hline 4 & 48 & $\mathrm{~F}$ & skull base & squamous cell carcinoma \\
\hline 5 & 69 & M & skull base & adenoid cystic carcinoma \\
\hline 6 & 68 & $\mathrm{~F}$ & skull base & adenoid cystic carcinoma \\
\hline 7 & 62 & $\mathrm{M}$ & skull base & multiple myeloma \\
\hline 8 & 37 & M & skull base & olfactory neuroblastoma \\
\hline 9 & 50 & $\mathrm{~F}$ & skull base & plasmacytoma \\
\hline 10 & 8 & $\mathrm{~F}$ & brain stem & anaplastic ependymoma \\
\hline 11 & 32 & $\mathrm{~F}$ & vertebral body & myxoid liposarcoma \\
\hline 12 & 10 & $\mathrm{M}$ & vertebral body & PNET \\
\hline 13 & 67 & M & vertebral body & adenocarcinoma \\
\hline
\end{tabular}

F: female, M: male, PNET: primitive neuroectodermal tumor.

graphy (CT) and magnetic resonance (MR) imaging were used for planning, and three-dimensional geometrical registration of the CT and MR imaging data was performed. All patients were treated with IMRT.

The planning target volume (PTV), which was defined as the area of the enhanced lesion with $3 \mathrm{~mm}$ margin, and OARs, such as the brain stem, eye balls, optic nerves, chiasma, and spinal cord, were delineated in the corresponding MR imaging and CT slices. Three OARs were chosen and numbered from 1 to 3 in order of distance from the lesion in each patient. OAR1 was closer to the PTV than the other OARs.

Both dynamic 3D-CRT and IMRT treatment plans were calculated for each patient by the same treatment planning system, Brain SCAN (BrainLAB $\mathrm{GmbH}$, Heimstetten, Germany). To eliminate interoperator variations, the same operator established all treatment plans. The same prescribed dose was determined in the dynamic 3D-CRT and IMRT plans to compare the two treatment plans for the same patient. The IMRT plan covered the entire PTV with higher than $95 \%$ of the prescribed dose (V95 was $100 \%$ ). The dynamic 3D-CRT plan covered the entire PTV with higher than $80 \%$ of the prescribed dose (V80 was 100\%). The percentage of the corresponding prescribed dose was calculated for the OARs to facilitate dosimetric comparison because each patient was treated with different prescribed doses.

To compare the dynamic 3D-CRT plan and the IMRT plan, we used dosimetric distribution and dose volume histogram (DVH) techniques. Dosimetric distribution is a two-dimensional contour map of 
delivered dose on some representative CT or MR imaging plane. The area inside the $80 \%$ contour line receives more than $80 \%$ of the prescribed dose. $\mathrm{DVH}$ is a three-dimensional dosimetric distribution in a graphical two-dimensional format, so DVH visualizes the volume not area. The vertical axis represents the percentage of total tissue volume that receives a dose more than a specified dose. The horizontal axis represents cumulative dose. Ideally, DVH displays $100 \%$ of the PTV receiving $100 \%$ of the prescribed dose and very low volumes of OARs receiving very low doses.

The homogeneity index (HI) was calculated using the following formula without using the prescribed
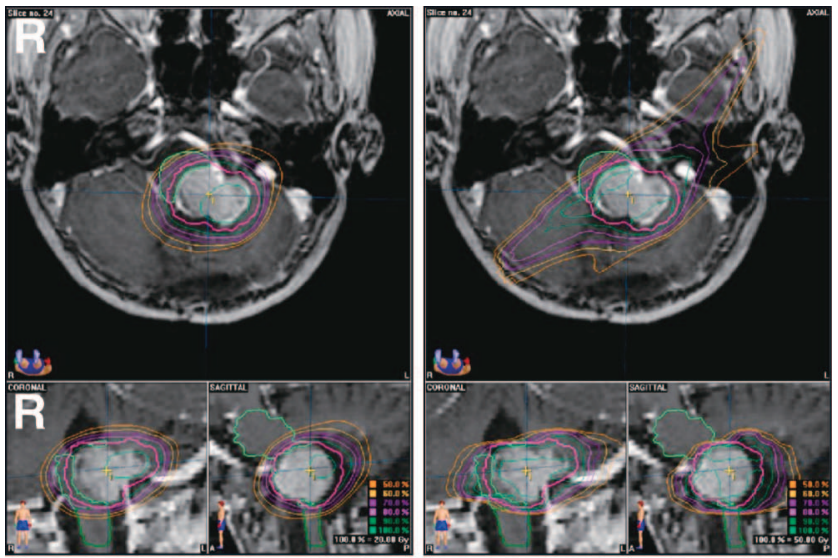

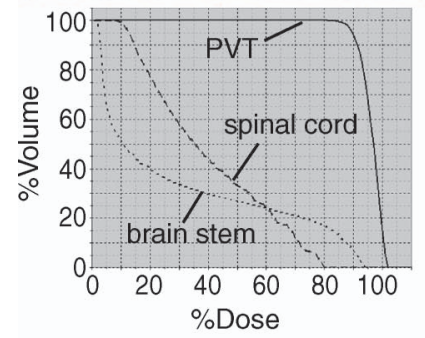

Dynamic 3D-CRT

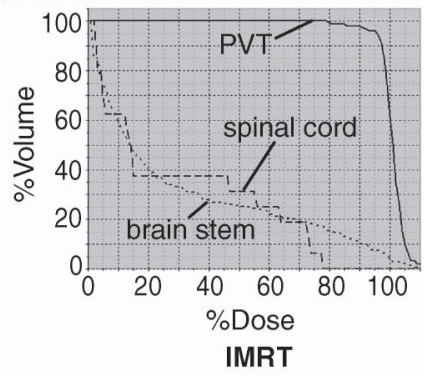

Fig. 2 Dosimetric distributions (upper row) and dose volume histograms (lower row) of the dynamic threedimensional conformal radiotherapy (3D-CRT) plan (left column) and the intensity modulated radiotherapy (IMRT) plan (right column) in representative Case 10. An 8-year-old girl presented with recurrent brain stem anaplastic ependymoma. Magnetic resonance images showed an oval-shaped tumor which compressed the brain stem into a concave form. In the dynamic 3D-CRT plan, the conformal dose was delivered to the planning target volume (PTV) but the brain stem and the spinal cord were not spared. In the IMRT plan, flat dosimetric distribution spared the brain stem and the spinal cord. Dose volume histogram of the IMRT plan showed dose reduction to the spinal cord without dose reduction to the PTV. dose: maximum dose within the PTV/minimum dose within the PTV. This formula is the simplest of several similar HIs. The HI is a useful tool to evaluate dose uniformity within the PTV and a value of HI close to 1 indicates better dose homogeneity in the treatment plan. ${ }^{6)}$

The conformity index (CI) was calculated using the following formula: $1+V_{\text {normal }} / \mathrm{V}_{\mathrm{PTV}}$. $\mathrm{V}_{\text {normal }}$ is the volume of the normal tissue and $\mathrm{V}_{\mathrm{PTV}}$ is the volume of the PTV receiving the indicated dose; $95 \%$ of the prescribed dose in the IMRT plan and $80 \%$ in the dynamic 3D-CRT in this study. Therefore, $V_{\text {PTV }}$ represented the entire PTV in both plans because we intended V95 as $100 \%$ in the IMRT plan and V80 as $100 \%$ in the dynamic 3D-CRT plan. A value of CI
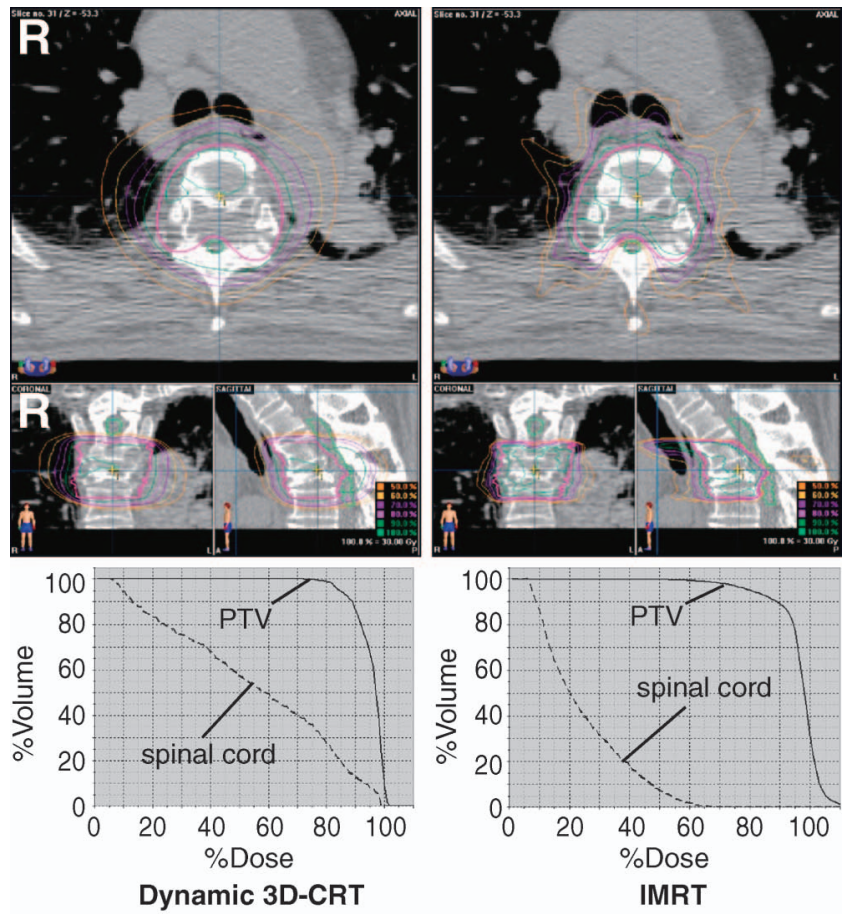

Fig. 3 Dosimetric distributions (upper row) and dose volume histograms (lower row) of the dynamic threedimensional conformal radiotherapy (3D-CRT) plan (left column) and the intensity modulated radiotherapy (IMRT) plan (right column) in representative Case 13. A 67-year-old man presented with vertebral body metastasis from lung cancer. Computed tomography scans showed that the concave-shaped tumor surrounded the spinal cord. The dosimetric distribution of dynamic 3DCRT was round and the spinal cord was not spared at all. The dosimetric distribution of IMRT was a conformal concave form corresponding with the planning target volume (PTV) and the spinal cord was spared. Dose volume histogram of the IMRT plan showed dose reduction to the spinal cord. 
close to 1 indicates better reduction of normal tissue irradiation ( $\mathrm{V}_{\text {normal }}$ is lower) in the treatment plan. ${ }^{4)}$

The data were analyzed by the Mann-Whitney U test. A probability value of $p<0.05$ was considered statistically significant. Statistical analyses were determined using StatView software (SAS Institute, Cary, North Carolina, USA).

\section{Results}

First, we show two representative cases in which IMRT was very effective.

Representative Case 10: An 8-year-old girl presented with recurrent brain stem anaplastic ependymoma. MR imaging showed an oval-shaped tumor which compressed the brain stem into a concave form. The brain stem, spinal cord, and optic nerve were defined as OARs, with the brain stem as OAR1. Dosimetric distributions are shown in Fig. 2. In the dynamic 3D-CRT plan, the conformal dose was delivered to the PTV but the brain stem and the spinal cord were not spared. In the IMRT plan, the flat dose distribution spared the brain stem and the spinal cord. DVH showed dose reduction to the spinal cord without dose reduction to the PTV. This patient was treated with IMRT using a prescribed dose of 50 Gy. MR imaging showed obvious tumor shrinkage. However, local recurrence was observed 9 months after IMRT treatment and the patient died 6 months after the recurrence. There was no complication due to IMRT.

Representative Case 13: A 67-year-old man presented with vertebral body metastasis from lung cancer. This lesion was impossible to treat with conventional radiotherapy because he had been already treated with conventional radiotherapy to the neighboring vertebral body with a total dose of $30 \mathrm{~Gy}$ in 10 fractions. The OAR of this patient was the spinal cord. CT showed that the concave-shaped tumor surrounded the spinal cord (Fig. 3). The dosimetric distribution of dynamic 3D-CRT was round and the spinal cord was not spared at all. We expected that this lesion would be difficult to treat with dynamic 3DCRT practically because of the high dose to the spinal cord. The dosimetric distribution of IMRT was a conformal concave form corresponding with the PTV and the spinal cord was spared. DVH showed dose reduction to the spinal cord. This patient was treated with IMRT using a prescribed dose of $30 \mathrm{~Gy}$. The patient had no local recurrence or radiological complication, such as paraparesis of the lower limbs, for 3.5 years.

Nine patients with skull base tumors were enrolled, 6 cases located at the maxillary sinus and the others
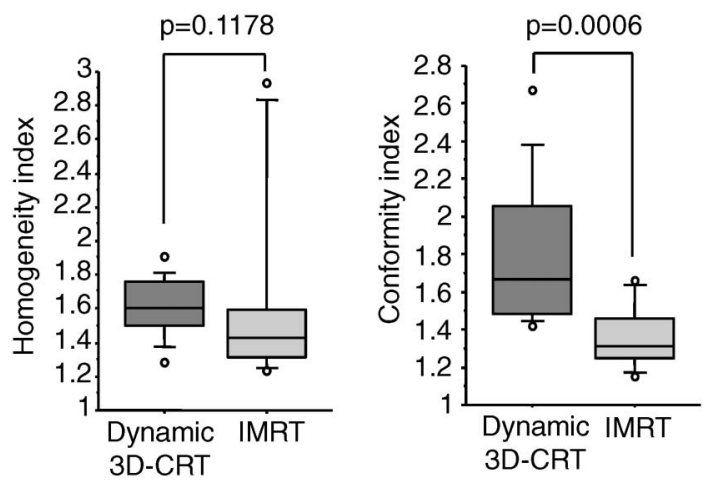

Fig. 4 Homogeneity index and conformity index of the dynamic three-dimensional conformal radiotherapy (3D-CRT) plan and the intensity modulated radiotherapy (IMRT) plan. In 11 of the 13 cases, the homogeneity index of the IMRT plan was lower than that of the dynamic 3D-CRT plan but the IMRT plan was not significantly superior to the dynamic $3 D-C R T$ plan $(p=0.1178)$. The conformity index of the IMRT plan (mean 1.358) was significantly better $(p=0.0006)$ than that of the dynamic 3D-CRT plan (mean 1.802). Statistical analyses were determined using StatView software.

at the orbit, the upper pharynx, and the cribriform plate. The OARs were usually the eye balls, optic nerves, optic chiasm, and brain stem. The optic nerve was most frequently designated as the OAR1. Almost all PTVs of skull base cases were not concave and sparing the OARs was easier than in the 2 representative cases. The most frequent disease of the skull base was maxillary squamous carcinoma. All 4 patients had new lesions and underwent chemotherapy and IMRT with a prescribed dose of $54 \mathrm{~Gy}$. All patients remained alive with mean overall survival of $1770 \pm 253$ days, although one patient experienced local recurrence. No radiological complication was observed.

The IMRT and dynamic 3D-CRT plans were compared for target dose homogeneity, target dose conformity, and mean dose to OARs. In 11 of the 13 patients, the HIs of the IMRT plans were lower than those of the dynamic 3D-CRT plans but the IMRT plan was not significantly superior to the dynamic 3D-CRT plan ( $p=0.1178)$ because the HIs of IMRT plans of only two cases were extremely close to 3 (Fig. 4). In those cases, the PTV attached and surrounded the OAR1 (one of the two cases was representative Case 13) and the minimum dose within the PTV was very low to decrease the dose to the OAR1. The CI of the IMRT plan (mean 1.358) was significantly better $(p=0.0006)$ than that of the dynamic 3D-CRT plan (mean 1.802) (Fig. 4). The percentage of the corresponding prescribed dose of the 

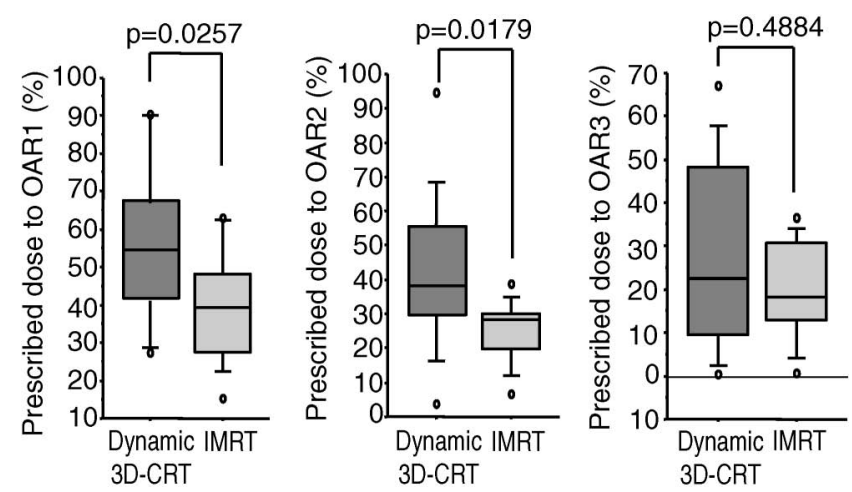

Fig. 5 Prescribed doses to the organs at risk (OARs) of the three-dimensional conformal radiotherapy (3D-CRT) plan and the intensity modulated radiotherapy (IMRT) plan. The percentage of the corresponding prescribed doses of the IMRT plan in the OAR1 (mean $41.0 \%$ ) and OAR2 (mean $25.5 \%$ ) were significantly lower than those of the dynamic 3D-CRT plan (mean $58.2 \%$ and $41.9 \%$, respectively). Statistical analyses were determined using StatView software.

IMRT plan in mean dose to the PTV (mean 98.8\%) was obviously higher $(p=0.0052)$ compared to that of the dynamic 3D-CRT plan (mean 96.3\%) (figure not shown). The percentage of the corresponding prescribed dose of the IMRT plan in the OAR1 (mean $41.0 \%)$ was significantly lower $(p=0.0257)$ than that of the dynamic 3D-CRT plan (mean 58.2\%) (Fig. 5 ). The percentage of the corresponding prescribed dose of the IMRT plan in the OAR2 (mean 25.5\%) was also lower $(p=0.0179)$ than that of the dynamic 3D-CRT plan (mean $41.9 \%$ ). However, there was no significant difference in the OAR3 between the plans $(\mathrm{p}=0.4884)$.

\section{Discussion}

The aim of radical radiotherapy is to deliver a high dose to the tumor target while minimizing the dose to surrounding normal tissues. Recent innovations of irradiation technique may meet these contradictory requirements. Stereotactic radiosurgery/radiotherapy (SRS/SRT) is one of these novel irradiation techniques. SRS/SRT was chiefly developed in recent years for dose escalation sparing the OARs, and can deliver much conformal doses to spherical tumors. For spheroidal tumors, the two-isocenter plan is more effective than the one-isocenter plan. ${ }^{11)}$ However, SRS/SRT is not suitable for irregularly shaped tumors. The multiple-isocenter plan can deliver a more conformal dose, but is more complex and the technique has limits. ${ }^{9}$ Dynamic 3D-CRT is one of the most excellent stereotactic radiothera- pies. $^{2,16)}$ Dynamic 3D-CRT with the MLC can deliver more conformal doses to the irregularly shaped tumors than SRS/SRT (Fig. 1), but sophisticated doses are still difficult to deliver to the concave form. In addition, SRS/SRT usually delivers a higher dose to the center of the PTV and the high dose spot over the prescribed dose is accrued within the PTV when the peripheral region receives a sufficient treatment dose.

IMRT is another advanced irradiation method without using stereotactic technologies, can modulate radiation intensity with MLC and deliver more homogeneous and conformal radiation doses using fixed beams based on inversely calculated planning (Fig. 1). The most important feature of IMRT is the excellent dose delivery conforming to irregularly shaped tumors, especially concave shaped tumors (representative Case 13). Our study showed that IMRT could deliver more homogeneous and conformal doses to irregularly shaped tumors than dynamic 3D-CRT, but the superior homogeneity was not statistically important because of sparing of the OAR surrounded by the PTV. In the treatment phase, the surgeon must determine whether the PTV has priority over the OAR or not. The decision is probably case-by-case.

Sparing of the OARs is very important as well as to treat the PTV with homogeneous and conformal dose. In our study, we intended that the entire PTV received higher than $95 \%$ of the prescribed dose in IMRT and $80 \%$ in dynamic 3D-CRT. Therefore, the percentage of the corresponding prescribed dose of the IMRT plan in the mean dose to the PTV was higher than that of the dynamic 3D-CRT plan. However, the receiving doses of the OARs were significantly lower in IMRT using the same planning. In OAR1 and OAR2, the differences in the dynamic 3D-CRT plan and the IMRT plan were significant but the difference in the OAR2 was greater than that in the OAR1. Therefore, the OAR adjoining the PTV is difficult to spare. In our case of brain stem tumor (representative Case 10), the lesion had compressed the brain stem into a concave form, but IMRT delivered a conformal dose to the PTV, sparing the spinal cord but not sparing the brain stem adequately. On the other hand, the difference in the OAR3 was not significant. Both dynamic 3D-CRT and IMRT plans could deliver treatment doses to the PTV sparing the OARs distant from the target.

Heavy charged particle radiotherapy is another promising treatment. Heavy charged particles, such as carbon ions, have excellent dose localizing properties compared with fast neutrons. The Bragg peak of the energy loss occurs immediately before the particles come to rest and the maximum depth of 
penetration of a charged particle beam can be adjusted by varying the energy. These features of the particles result in excellent dose conformity. Comparisons of heavy charged particle radiotherapy and IMRT showed that heavy charged particle radiotherapy delivered a more sophisticated conformal dose to the target than IMRT. ${ }^{15)}$ However, few establishments worldwide have heavy ion medical accelerator equipment and the cost performance is usually poor. Presumably heavy charged particle radiotherapy will not become common as standard radiotherapy at the present time.

All of our 13 patients were treated with IMRT, and radiosensitive tumors were successfully controlled for several years with sufficient sparing of OARs. However, in radioresistant tumor such as anaplastic ependymoma, we could not inhibit tumor proliferation using IMRT with the usual irradiation dose. Dose escalation to the PTV improves local control of the tumors, ${ }^{7,11)}$ but also increases the risk of normal tissue complication. Safe irradiation requires delivery of homogeneous and conformal dose to the PTV and minimum doses to the OARs. Although IMRT is one of the best radiotherapies for dose escalation, the complex treatment plan and long treatment time are problems. In the choice of irradiation modality, we have to consider the cost/benefit ratio, and IMRT is suitable for irregularly shaped tumors close to OARs.

In this study, dynamic 3D-CRT and IMRT were compared with regard to dose distribution and sparing of OARs. IMRT was superior to dynamic 3DCRT for irradiation of irregularly shaped tumors near the OARs. Therefore, dose escalation using IMRT will improve local control of radioresistant tumors in the future.

\section{References}

1) Bahri S, Flickinger JC, Kalend AM, Deutsch M, Belani CP, Sciurba FC, Luketich JD, Greenberger JS: Results of multifield conformal radiation therapy of nonsmall-cell lung carcinoma using multileaf collimation beams. Radiat Oncol Investig 7: 297-308, 1999

2) Dearnaley DP, Khoo VS, Norman AR, Meyer L, Nahum A, Tait D, Yarnold J, Horwich A: Comparison of radiation side-effects of conformal and conventional radiotherapy in prostate cancer: a randomized trial. Lancet 353: 267-272, 1999

3) Ding M, Newman F, Chen C, Stuhr K, Gaspar LE: Dosimetric comparison between 3DCRT and IMRT using different multileaf collimators in the treatment of brain tumors. Med Dosim 34: 1-8, 2009

4) Feuvret L, Noël G, Mazeron JJ, Bey P: Conformity index: a review. Int J Radiat Oncol Biol Phys 64: 333-342, 2006
5) Hanks GE, Hanlon AL, Pinover WH, Horwitz EM, Schultheiss TE: Surgical advantage for prostate cancer patients treated with high-dose three-dimensional conformal radiotherapy. Cancer J Sci Am 5: 152-158, 1999

6) Kam MK, Chau RM, Suen J, Choi PH, Teo PM: Intensity-modulated radiotherapy in nasopharyngeal carcinoma: dosimetric advantage over conventional plans and feasibility of dose escalation. Int J Radiat Oncol Biol Phys 56: 145-157, 2003

7) Khoo VS, Oldham M, Adams EJ, Bedford JL, Webb S, Brada M: Comparison of intensity-modulated tomotherapy with stereotactically guided conformal radiotherapy for brain tumors. Int J Radiat Oncol Biol Phys 45: 415-425, 1999

8) Kong FM, Ten Haken RK, Schipper MJ, Sullivan MA, Chen M, Lopez C, Kalemkerian GP, Hayman JA: High-dose radiation improved local tumor control and overall survival in patients with inoperable/unresectable non-small-cell lung cancer: long-term results of a radiation dose escalation study. Int $\mathrm{J}$ Radiat Oncol Biol Phys 63: 324-333, 2005

9) Kramer BA, Wazer DE, Engler MJ, Tsai JS, Ling MN: Dosimetric comparison of stereotactic radiosurgery to intensity modulated radiotherapy. Radiat Oncol Investig 6: 18-25, 1998

10) Kwong DL, Pow EH, Sham JS, McMillan AS, Leung LH, Leung WK, Chua DT, Cheng AC, Wu PM, Au GK: Intensity-modulated radiotherapy for early-stage nasopharyngeal carcinoma: a prospective study on disease control and preservation of salivary function. Cancer 101: 1584-1593, 2004

11) Mignano JE, Engler MJ, Tsai JS, Wazer DE: Comparison of radiobiologic modeling for one- and twoisocenter dose distributions applied to ellipsoidal radiosurgery targets. Int J Radiat Oncol Biol Phys 49: 833-837, 2001

12) Nieder C, Berberish $W$, Nestle U, Niewald M, Walter K, Schnabel K: Relation between local result and total dose of radiotherapy for brain metastases. Int J Radiat Oncol Biol Phys 33: 349-355, 1995

13) Sandler HM, Perez-Tamayo C, Ten Haken RK, Lichter AS: Dose escalation for stage C (T3) prostate cancer: minimal rectal toxicity observed using conformal therapy. Radiother Oncol 23: 53-54, 1992

14) Sankaranarayanan V, Ganesan S, Oommen S, Padmanaban TK, Stumpf J, Ayyangar KM: Study on dosimetric parameters for stereotactic radiosurgery and intensity-modulated radiotherapy. Med Dosim 28: 85-90, 2003

15) Schulz-Ertner D, Nikoghosyan A, Didinger B, Karger $\mathrm{CP}$, Jäkel O, Wannenmacher M, Debus J: Treatment planning intercomparison for spinal chordomas using intensity-modulated photon radiation therapy (IMRT) and carbon ions. Phys Med Biol 48: 2617-2631, 2003

16) Singh IR, Brindha S, Ravindran BP, John S, Rajshekhar V, Rangad FV, Roul RK: Development of collimator insert for linac based stereotactic irradiation. 
Phys Med Biol 44: 223-231, 1999

17) Teh BS, Mai WY, Grant WH 3rd, Chiu JK, Lu HH, Carpenter LS, Woo SY, Butler EB: Intensity modulated radiotherapy (IMRT) decreases treatment-related morbidity and potentially enhances tumor control. Cancer Invest 20: 437-451, 2002

18) Tsien C, Gomez-Hassan D, Ten Haken RK, Tatro D, Junck L, Chenevert TL, Lawrence T: Evaluating changes in tumor volume using magnetic resonance imaging during the course of radiotherapy treatment of high-grade gliomas: Implications for conformal dose-escalation studies. Int Radiat Oncol Biol Phys 62: 328-332, 2005

19) Wu VW, Kwong DL, Sham JS: Target dose conformity in 3-dimensional conformal radiotherapy and in- tensity modulated radiotherapy. Radiother Oncol 78: 78-79, 2005

20) Zelefsky MJ, Leibel SA, Kutcher GJ, Fuks Z: Threedimensional conformal radiotherapy and dose escalation: where do we stand? Semin Radiat Oncol 8: 107-114, 1998

Address reprint requests to: Yuzo Hasegawa, MD, Division of Neurological Surgery, Chiba Cancer Center, 666-2 Nitona-cho, Chuo-ku, Chiba, Chiba 260-8717, Japan. 